\title{
Performance degradation estimation of robot in highly radioactive environment: Servo Motor Control
}

\author{
Shimeng Li ${ }^{1}$, Hongfei Wang ${ }^{1}$, Yuan F. Zheng ${ }^{1}$, and Lei (Raymond) $\mathrm{Cao}^{2}$ \\ ${ }^{1}$ Electrical and Computer Engineering, The Ohio State University, Columbus, Ohio 43210 USA \\ ${ }^{2}$ Mechanical Engineering, The Ohio State University, Columbus, Ohio 43210 USA
}

\begin{abstract}
For robot application in highly radioactive environment, the possible radiational damage to the components must be evaluated. Particularly, our work discussed in this paper chooses servo motor as the research object, considering motor control issues which is more realistic in practical robot application. We study the performance degradation of the robot servo motor by introducing the radiation effect on the specific motor constant in the motor model, establish a PID control scheme for motor speed control, and simulate the radiation-induced performance degradation.
\end{abstract}

\section{Introduction and Review}

Being aware of the high risk for humans working in the highly radioactive environment such as the nuclear power plants of Chernobyl and Fukushima Daiichi) for rescuing, repairing, and maintenance tasks, robots are adopted as good substitution for completing the complex missions in high-risk areas because of the intelligence, mobility and accuracy of the robots [1]. However, this idea has not yet been taken into practice since robot components can be damaged and loses functionality due to high dose radiation in the accident area [2,3]. Research concerns on radiation-hardened robot components and applications have been ever since 2007, when NASA carried on the Radiation Hardened Electronics for Space Environments (RHESE) project [4] for developing radiation hardened electronic components. In 2013, the DARPA grand robotic challenge was aiming at completing particular tasks that humanoid robots can do in the nuclear accident area [5].Eight tasks were established, simulating the situations that a rescuing robot may encounter in a nuclear power plant in accident.

One of the competing robots in the DARPA challenge, Hubo+, is shown in Figure 1. Hubo+ is an adult-sized humanoid robot platform with 38 Degrees of Freedoms (DOF), each of which consists of one servo motor and one harmonic drive as speed reducer. The servo motor is controlled by a joint motor control board, and all the boards receive command from an inside processor which serves as the main controller. The environmental feedback data retrieved from the sensors and cameras are also processed in the main controller. According to previous studies, the electronic components in the robot system, including the controllers, cameras, and sensors, have been proved vulnerable under radioactive environments and studied on how the performance will degrade after irradiation [6-8]. However, the radiation effect on the performance of the mechanical components, like the servo motor and speed reducer has never been well studied before.

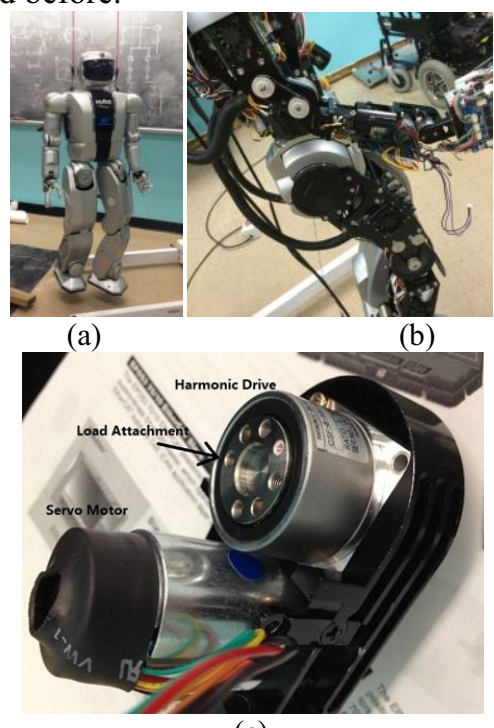

(c)

Figure 1. Hubo + Humanoid Platform (a) close view (b) and joint view(c).

Since 2012, we have been working on the research of radiation sensitivity of robot components. Our research focuses on the harmonic drive and brushless DC motor (BLDC). The harmonic drive is an ideal component for robots which allows large speed reduction ratio in compact size and light weight taking advantage of flexible material; the BLDC motor, on the other hand, 
gets rid of brushes and can be controlled sensorlessly so that there is no brush commutation loss during operation, and noises are significant reduced. Our research on the harmonic drive [9-11] has proved that these effects are very little. As for the BLDC motor, since it is impossible to irradiate the whole component because of potential risks of radioactivation, we irradiated the motor magnet only to see the radiation influence, and simulated the motor performance variation based on an ideal BLDC motor model introducing the influence, obtaining the result that the BLDC will be degraded in power and possibly be out of work caused by the "magnetic trap" jerking effect [12].

From our previous studies, we can draw a conclusion that the motor could be affected to a much more significant extent than the harmonic drive. Therefore, the follow-up work we present in this paper will be primarily based on the radiation induced degradation on the servo motor. We will assume an appropriate working condition for a robot servo, build up a complete servo motor control scheme rather than the simple PWM input in [12], illustrate more detailed modeling work, and analyze the system response simulated in MATLAB.

\section{System Modeling and Control}

\subsection{System configuration}

The robot model we will discuss in this paper is shown in Figure 2. Comparing with the humanoid robot like Hubo+, a wheeled robot is much focused on the motor which drives the wheels. The configuration of the robot is defined in Table 1. Ignoring the motions of the robot arms, the model can be viewed as simple as a wheeled vehicle driven by one servo motor. Our paper will focus on the control of this single DOF.

For the convenience of our modelling work, we choose the U12M4T servo disc motor as our object of simulation. The parameters of the motor is shown in Table 2 .

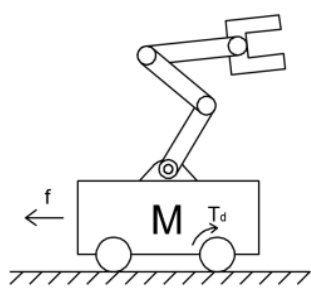

Figure 2. A wheeledmobile robot.

Table 1.Vehicle robot configuration.

\begin{tabular}{|c|c|c|c|}
\hline Parameter & Index & Value & Measurement \\
\hline Weight & $M$ & 10 & $\mathrm{~kg}$ \\
\hline Max Speed & $v_{\max }$ & 0.5 & $\mathrm{~m} / \mathrm{s}$ \\
\hline $\begin{array}{c}\text { Max } \\
\text { Acceleration }\end{array}$ & $a_{\max }$ & 0.2 & $\mathrm{~m} / \mathrm{s}^{2}$ \\
\hline $\begin{array}{c}\text { Wheel } \\
\text { Radius }\end{array}$ & $r_{w}$ & 0.1 & $\mathrm{~m}$ \\
\hline
\end{tabular}

Table 2. Servo motor parameters*.

\begin{tabular}{|c|c|c|c|}
\hline Parameter & Index & Value & Measurement \\
\hline Peak Torque & $T_{p}$ & 852 & $\mathrm{~N}^{*} \mathrm{~cm}$ \\
\hline $\begin{array}{c}\text { Nominal } \\
\text { Power }\end{array}$ & $P$ & 254 & $\mathrm{~W}$ \\
\hline $\begin{array}{c}\text { Nominal } \\
\text { Voltage }\end{array}$ & $U_{n}$ & 40.4 & $\mathrm{~V}$ \\
\hline $\begin{array}{c}\text { Nominal } \\
\text { current }\end{array}$ & $I_{m}$ & 8.71 & $\mathrm{~A}$ \\
\hline $\begin{array}{c}\text { Idle Speed } \\
\text { Rotor Inertia }\end{array}$ & $n_{0}$ & 6000 & $\mathrm{rpm}$ \\
\hline $\begin{array}{c}\text { Magnet } \\
\text { Remainance }\end{array}$ & $B_{r}$ & 1.2 & $\mathrm{kgcm}$ \\
\hline $\begin{array}{c}\text { Terminal } \\
\text { resistance }\end{array}$ & $R$ & 0.75 & $\Omega$ \\
\hline $\begin{array}{c}\text { Terminal } \\
\text { inductance }\end{array}$ & $L$ & $<0.1$ & $\mathrm{mH}$ \\
\hline
\end{tabular}

* The parameters in this table can be found on http://www.kollmorgen.com/en-us/products/motors/brushdc/servo-disc/_literature/servodisc_catalog_en-us_2003.pdf

\subsection{Radiation demagnetization modeling}

A regular motor dynamic model can be described as

$$
\left\{\begin{array}{c}
u=\left(R+\frac{d}{d t} L\right) i+e \\
\tau_{e}=e i / \omega \\
\frac{d \omega}{d t}=\left(\tau_{e}-\tau_{\text {load }}-b \omega_{r}\right) / j
\end{array}\right.
$$

in which $u$ is the applied input voltage, $i$ the current, $e$ the motor back-emf voltage, $j$ the moment of inertia, $b$ the damping coefficient, $\omega$ the motor angular velocity, $\tau_{e}$ the output torque, and $\tau_{\text {load }}$ the equivalent torque load.

The electromagnetic equation of motor back-emfof one coil can be described as a function of motor rotation speed according to the law of induction [12]:

$$
\begin{aligned}
e=-\frac{d \Psi}{d t} & =\omega \cdot\left(-\frac{d \Psi}{d \theta}\right)=\omega \cdot K_{b} \\
\text { and } \Psi & =N_{\text {turns }} \sum_{i=1}^{m} \int_{\alpha_{i}}^{\alpha_{i}+\alpha_{l}} B(\theta, \alpha) d \alpha .(2)
\end{aligned}
$$

where $N_{\text {turns }}$ is the number of turns in each armature, $m$ the number of armatures in a single phase, $\alpha_{i}$ the relative starting position of thecoil, and $\alpha_{l}$ the length of the coil, $B(\theta, \alpha)$ is a scalar function of rotor rotation $\theta$ and stator relative position $\alpha$ representing the B-field intensity on the stator surface.

Normally $d \Psi / d \theta$ is approximately periodic square wave function of $\theta$, and $K_{b}$ can be considered as a constant, which is called the motor back-emf constant or motor velocity constant. However for motor exposed to the radiation, the stator surface flux will be disturbed. The magnetic flux distribution can be modelled as a periodic function of angular position in the 1-D space [14]. In the electromagnetic model of BLDC motor, only the radial component is considered in the back-emf voltage calculation. The radiation causes a permanent change to the plant model, which is determined by the 
total absorbed dose by the magnet, the energy of radiation particle, and the distribution of radiation absorption of the magnet. The latter causes the reduction of the B-field intensity, i.e., demagnetization.Among those 3 factors, the first 2 has already been proved to have a considerable positive relationship with the total demagnetization [15]; the third factor, although has been observed in our previous experiment, has too many unpredictable and uncontrollable features, so we assume it to be an "inherent noise component" which is random in our model as [12]:

$$
\begin{gathered}
\delta=\frac{\Delta B}{B_{0}}=\frac{B_{0}-B}{B_{0}}=k \tilde{d}(\theta) \\
\text { and } \tilde{d}(\theta) \sim \widetilde{N}\left(\mu, \sigma_{a}^{2}\right)
\end{gathered}
$$

where $k$ is the power coefficient determined by the power of radiation source, $\tilde{d}(\theta)$ is an random B-field intensity reduction ratio following the distribution $\widetilde{N}\left(\mu, \sigma_{a}^{2}\right)$, in which $\mu_{e}$ is the average reduction ratio, $\sigma_{a}^{2}$ the variance of the same parameter.Our research in [16] has shown that the value of $d \Psi / d \theta$ always vary by a greater extent than the magnetic flux density $B$. Therefore, the motor back-emf constant $K_{b}$, will nolonger be a constant, but a number varying with the motor angular position.

Our study should be based on an obvious fact that the electronic driving circuits (if well protected from the radiation) will not know if the motor is irradiated and the magnet is demagnetized. So it will continue the original operation. As a result, we obtain different results if we give the same open-loop pulse-width-modulation (PWM) input to the "unirradiated motor plant model" and the "irradiated motor plant model". The latter will further show different performance whenthe radiation absorption by the motor is to different extent. However, for a servo motor on a robot, it is certain that we are able to take advantage of the sensory feedbacks, to realize control schemes like torque (current) control, speed control, and position control. For our study discussed in this paper, we still claim that the scheme and parameters of motor controller, and the sensory feedback data do not change with the state of motor plant "irradiated" or "unirradiated".

Particularly in this paper, we do not consider the possible commutation error as illustrated in [12], which is described as "magnetic trap" phenomenon, causing the motor jerk and quit working. This is because a servo motor is not necessarily a brushless one, and unlike the BLDC motor, the brushed DC motor does not have a mechanism of phase shifting control and the brushed commutation mechanism of motor armature currents cannot be influenced by radiation. Therefore, we focus on conditions where "magnetic trap" does not occur, and study how the change of motor velocity constant $K_{b}$, will affect the motor performance under properly controlled pattern of the radiation.

Normally the value of $K_{b}$ is approximated by the mean value of $-d \Psi / d \theta$ in the section of coil activation, as is shown in Figure 3. We take the data within the angular section between the two cursors as a sample. These data demonstrate little variation and can be considered as the constant $K_{b}$.

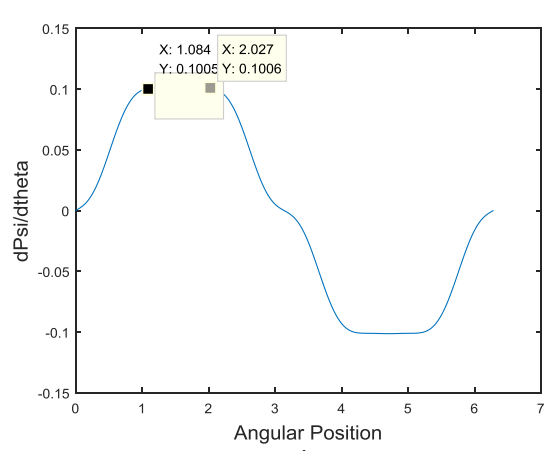

Figure 3. A typical pattern of $d \Psi / d \theta$ in one phase of BLDC motor. The constant portion is used for the value of $K_{b}$.

Then we set $k=5, \mu=0.03, \sigma_{a}=0.05$ in (3), and calculate the $d \Psi / d \theta$. The result is shown in Figure 4. We can observe that $d \Psi / d \theta$ within the same angular section is varying in a random pattern around $K_{b}$ in Figure 4 (a), and the probability of variationgenerally goes down while the extent of variation getting larger. It should be noted that the variation probability does not necessarily follow an exact Gaussian distribution, from a bunch of results simulated other than what is shown in Figure 4.

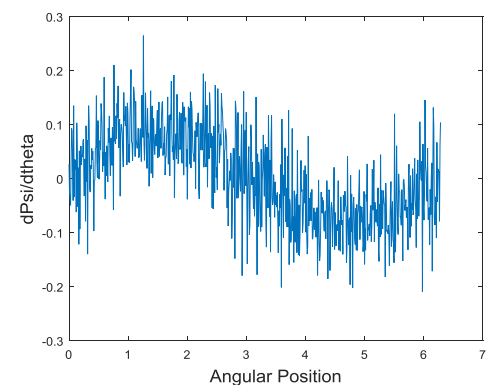

(a)

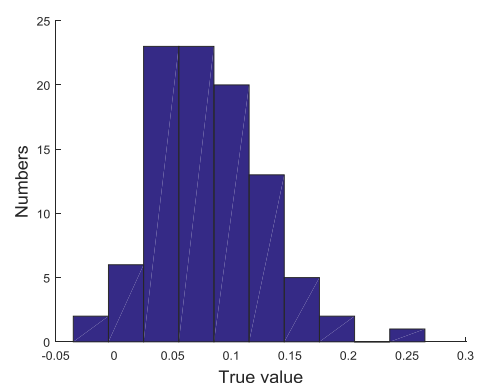

(b)

Figure 4. Irradiated pattern (a) and histogram (b) of $d \Psi / d \theta$ of the motor $\left(k=5, \mu=0.03, \sigma_{a}=0.05\right)$.

\subsection{Control design}

Generally, PID controller is used for many control problems because of its simple structure and easy implementation. For controlling a robot servo motor, the most usual application is position control and speed control. We also know that normally the motor torque control is achieved by maintaining the armature current knowing that the motor torque coefficient can be viewed as a constant. However motor behaviors will be changed after introducing radiation factors to the motor plant.

The position controller is designed as an outer loop of speed control, with a maximum speed defined; and the speed controller and the current controller are designed on two parallel loops, between which only one of the two 
can be activated at one time. As we are dealing with only on the wheel of the vehicle robot, we focus on the speed control of the servo motor.

\section{Simulation and Analysis}

The simulation works are performed in MATLAB. The system sampling frequency is set at $200 \mathrm{~Hz}$. Since no practical experiment data is referred in this paper, we ignore the circuit delay, the environmental disturbance, and the sensor error. This allows us to have a more direct and clear view on the radiation impact on the motor servo system. Assume that the constant load torque is $f=9.8 \mathrm{~N}$. The reference speed of the vehicle robot is set at $0.3 \mathrm{~m} / \mathrm{s}$.

As we have already illustrated in the previous section, although it is very difficult to grasp the pattern of $K_{b}$ variation after irradiation, it is possible for us to confirm a region of $K_{b}$ variation where the true value of $K_{b}$ is very probable to locate in. The distribution of $K_{b}$ can also follow the histogram in Figure 4 (b) since we are using real motor parameters as reference in MATLAB simulation.

The motor speed PID control functions in the time domain can be described as

$$
\begin{gathered}
\left\{\begin{array}{r}
v(t)=u(t) * v_{\text {nom }} \\
\dot{\omega}(t)=\frac{\frac{K_{b}}{R} v(t)-\tau_{\text {load }} / n-\left(b+\frac{K_{b} K_{\tau}}{R}\right) \omega(t)}{J}
\end{array}\right. \\
\text { and } u(t)=\begin{array}{r}
k_{p}\left(\omega_{d}-\omega(t)\right)-k_{d} \dot{\omega}(t)+k_{i} \int_{0}^{t} \omega(t) d t=D \\
0<D \leq 1 \\
0 \\
1
\end{array} \quad \begin{array}{r}
D>1 \\
D>1
\end{array}
\end{gathered}
$$

in which $v_{\text {nom }}$ is the nominal voltage of the motor, $n$ the speed reduction ratio, $\omega(t)$ the motor speed, $K_{\tau}=K_{b}$. since we are using SI units for all values, the control signal $u(t)$ is defined as PWM duty cycle varying from 0 to $1 . k_{p}, k_{i}$, and $k_{d}$ are PID control gains, $\omega_{d}$ is the desired motor speed.

When the radiation influence has been involved, $K_{b}$ will no longer be constant but a periodic signal varying with the motor position. So the first part of (4) becomes

and

$$
\left\{\begin{array}{c}
v(t)=u(t) * v_{\text {nom }} \\
\dot{\omega}(t)=\frac{\frac{\widetilde{K}_{b}(\Theta)}{R} v(t)-\tau_{\text {load }} / n-\left(b+\frac{\widetilde{K}_{b}{ }^{2}(\theta)}{R}\right) \omega(t)}{J}
\end{array}\right.
$$

$$
\begin{gathered}
\Theta=\int_{0}^{t} \omega(t) d t-2 m \pi \\
m=. .,-2,-1,0,1,2, . . \quad 0 \leq \Theta<1
\end{gathered}
$$

As we have illustrated before, the system itself does not know whether the motor is irradiated or not, so the same control reference are provided to the unirradiated and irradiated motors, respectively. We set reduction ratio $n=100$, control gains $k_{p}=0.0025, k_{i}=0.0032$, and $k_{d}=0.0004$, with the radiation parameters $k=5$, $\mu=0.03, \sigma=0.05$, same as in Figure 4. Then we simulate the response of the speed control which is shown in Figure 5.

What we have observed is that: the speed control seems to be functioning normally on the irradiated motor; while the voltage/speed ratio of the motor has increased and the current/torque ratio has decreased, both by a significant scale. This variationis predictable because in Figure 4 , we have seen from the histogram that the average $K_{b}$ has decreased significantly. The noises during operation does not exist in the simulation because the motor is rotating in a relatively high speed due to the existence of motor speed reducer.

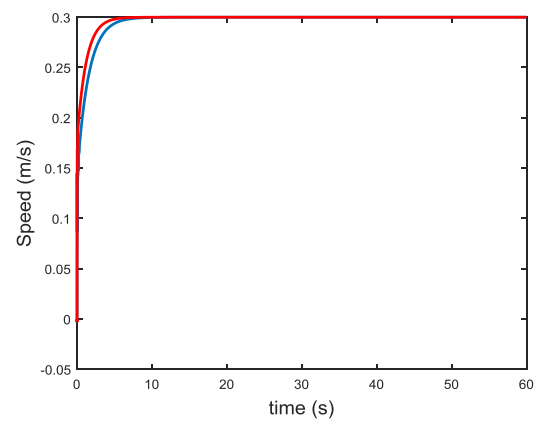

(a)

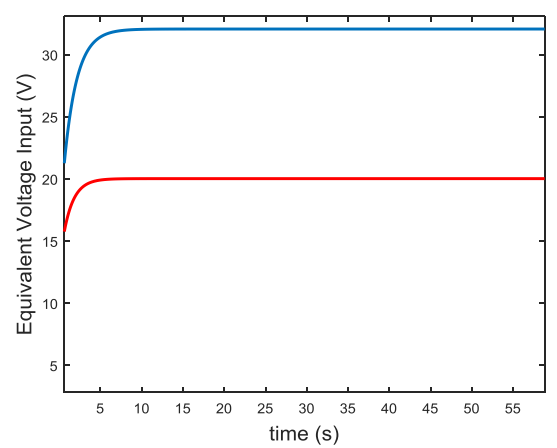

(b)

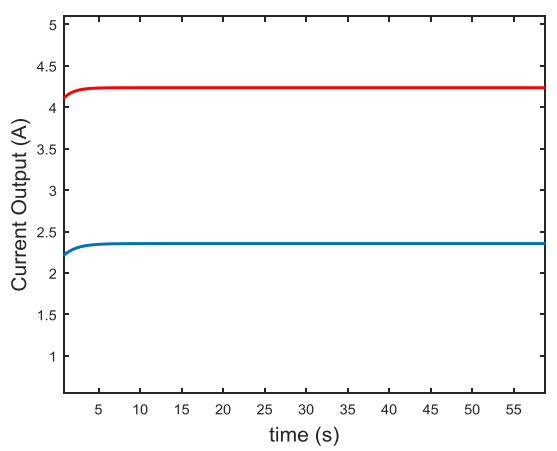

(c)

Figure 5. Simulation results of unirradiated (blue plot) and irradiated servo motor (red plot) $(k=5)$.

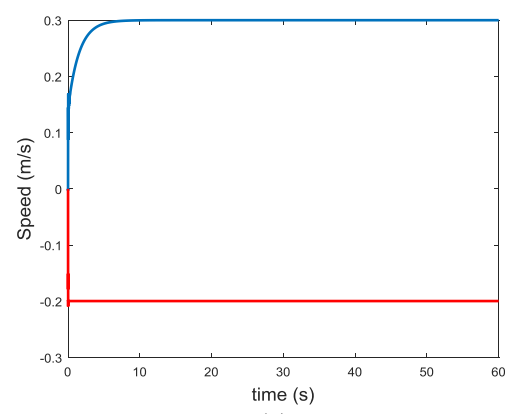

(a) 


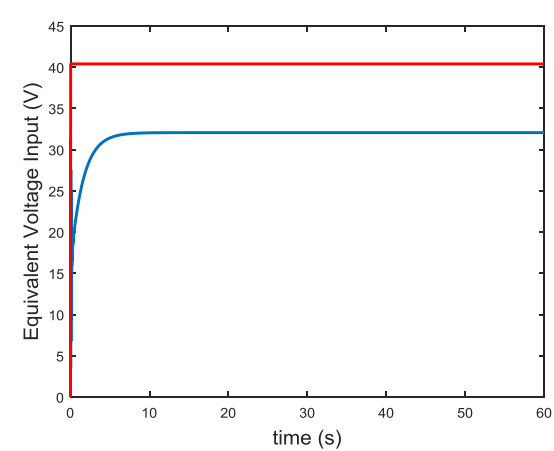

(b)

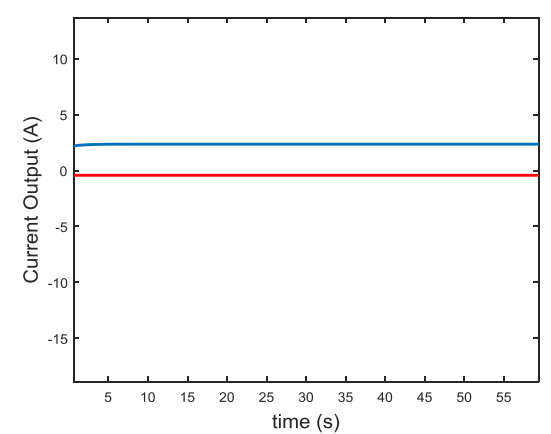

(c)

Figure 6. Simulation results of unirradiated (blue plot) and irradiated servo motor (red plot) $(k=20)$.

The radiation parameters, $\mu$ and $\sigma$, are parameters related to the average particle energy and spacial distribution, respectively, which are difficult to control in reality. Therefore, we increase $k$, which has a positive relationship with the radiation dose, to see how the motor performance will change when the radiation dose increases. The simulation results are shown in Figure 6 . Comparing to Figure 5, the motor speed control has failed, current dropped below zero, and the input voltage remains constantly maximum. And from the previous result, we can conclude that since $K_{b}$ has decreased in a greater extent, the motor itself cannot generate enough torque to overcome the load torque. In this condition, the motor is back driven by the constant load torque, and we can easily imagine that if a coulomb frictional load, of which the maximum is over the motor capability, is applied, the motor will be stalled with no rotation so there will be more risk of over-current damage to the motor.

\section{Conclusion}

In this paper, we have established a servo motor control scheme for the servo motor in a vehicle robot, and simulated the performance of speed control for the irradiated and unirradiated motor models. We model the radiation influence as a disturbance to the motor backemf constant, and simulated the system response with PID control. The result shows that with PID motor speed control at high speed will eliminate the noises we have observed in previous research; on the other hand, since $K_{b}$ has decreased, the voltage/speed ratio of the motor has increased while the current/torque ratio has decreased, thus inducing greater risk that the motor could not generate enough torque, and when the motor is stalled, it is easier to get damaged by over-current.

\section{Acknowledgement}

This work was supported by the U.S. Defense Threat Reduction Agency under Contract HDTRA1-13-1-0012.

\section{References}

1. I. Karl, and S. Dubowsky, The 26th ASME Biennial Mechanisms and Robotics Conference, 2000. (DETC 2000)

2. J. Wyss, D. Bisello, and D. Pantano, Nuclear Instruments and Methods in Physics Research Section A: Accelerators, Spectrometers, Detectors and Associated Equipment, 462(3). (2001)

3. P.E. Dodd, and L.W. Massengill, IEEE Transactions on Nuclear Science, 50(3). (2003)

4. A. Keys, J.H. Adams, and J.D. Cressler, et al., Technology, 4. (2007)

5. P. Gill, and J. Manzo, Robotics \& Automation Magazine, 20(2). (IEEE 2013)

6. K. Nagatani, S. Kiribayashi, and Y. Okada, et al., International Symposium on Safety, Security, and Rescue Robotics, Kyoto, Japan, (IEEE 2011)

7. Y. Yao, F. Dai, and R.C. Jaeger, et al., IEEE Transactions on Industrial Electronics, 55(7). (2008)

8. G. Piolain, F. Geffard, and A. Coudray, et al., 1st International Conference on Applied Robotics for the Power Industry, Montreal, Canada. (IEEE 2010)

9. S. Li, Y. F. Zheng, and A. J. Samin, et al., International Conference on Mechanical Design, Manufacture and Automation Engineering, Phuket, Thailand. (2014)

10. S. Li, Y. F. Zheng, and L. Cao, International Conference on Mechatronics and Automation, Tianjin, China. (IEEE 2014)

11. S. Li, Y. F. Zheng, and J. Qiu, et al., International Journal of Mechatronics and Automation (in press).

12. S. Li, Y. F. Zheng, and J. Qiu, et al., International Journal of Mechatronics and Automation (accepted).

13. P. Pillay, K. Ramu, IEEE Transactions on Industry Applications, 25(2). (1989)

14. P. Kumar, P. Bauer, IEEE Transactions on Magnetics, 44(10), (2008)

15. J. Alderman, P.K. Job, and R.C. Martin, et al., Nuclear Instruments and Methods in Physics Research Section A: Accelerators, Spectrometers, Detectors and Associated Equipment, 481(1). (2002)

16. S. Li, Y. F. Zheng, and J. Qiu, et al., International Conference on Electro/Information Technology, Dekalb, IL, USA. (IEEE 2015) 